\title{
IL12/IL23 Inhibition in the Treatment of Psoriatic Arthritis
}

\author{
Mahta Mortezavi, $M D^{*}$ \\ Christopher Ritchlin, MD, MPH
}

\author{
Address \\ *Division of Allergy, Immunology and Rheumatology, University of Rochester \\ Medical Center, 601 Elmwood Ave.B0X 695, Rochester, NY 14642, USA \\ Email:mahta_mortezavi@urmc.rochester.edu \\ Published online: 16 April 2015 \\ (C) Springer International Publishing AG 2015
}

This article is part of the Topical Collection on Spondyloarthritis and Psoriatic Arthritis

Keywords Psoriasis • Psoriatic arthritis · IL12 • IL17 • IL22 • IL23 • p40 • p19

\section{Opinion statement}

Psoriatic arthritis (PsA) is a potentially crippling systemic inflammatory joint disease with a heterogeneous clinical presentation that may include articular inflammation, enthesitis, dactylitis, abnormal bone turnover, and plaque psoriasis. Until very recently, patients with PsA had very few treatment options available to them and suffered immense physical and social burden from this disease. The use of anti-TNF agents offered some hope to these patients as a percentage of them found improvement of their psoriasis and psoriatic arthritis. However, over time, the biggest issue with anti-TNF drugs for PsA has been the findings that up to $50 \%$ of patients do not respond to or do not tolerate these agents. Ustekinumab (UST) was one of the first agents to offer an alternative mechanism for the treatment of psoriasis and PsA, by blocking the IL12 and IL23 cytokines. The use of this medication relates directly to our improved understanding of the pathophysiology of psoriasis and PsA as being driven by Th1 and Th17 cells. In the PSUMMIT II study, patients naive to anti-TNF agents responded better to UST than patients who were previously treated with those medications. These data indicate that a subset of PsA patients manifest persistently active disease refractory to agents that target alternative immune inflammatory pathways not directly related to TNF. These findings have also been observed in RA. Thus, targeting more than one molecule with an antibody that recognizes two different targets or combination biologics may be required to drive higher treatment responses or even remission. Of course, a major obstacle aside from safety is cost; on average UST, the 90-mg dose can be significantly more expensive compared to anti-TNF agent. However, as new safe and efficacious agents become available for the treatment of psoriasis and PsA, our treatment algorithm for the treatment of PsA will need to be re-evaluated. 


\section{Introduction}

Psoriatic arthritis (PsA) is a systemic inflammatory joint disease with a heterogeneous clinical presentation that may include peripheral or axial arthritis with altered bone phenotypes, enthesitis, dactylitis, and plaque psoriasis. Psoriasis is a relatively common disease, affecting about $2 \%$ of the general population; up to $25 \%$ of these patients develop PsA about 10 years after the development of skin disease [1]. Increasing knowledge about the pathophysiology of psoriasis and PsA has revealed that the diverse disease manifestations observed in these disorders may share common immunologic and inflammatory pathways. In the case of psoriasis, antigen driven activation of dendritic cells by interferon $\alpha$ (IFN- $\alpha$ ) promotes aTh1 and Th17 cell response in lymph nodes draining the skin $[2,3]$. These cell subpopulations release a variety of cytokines including interleukin (IL) 17, IL21, IL22, and IL23 and tumor necrosis factor $\alpha$ (TNF- $\alpha$ ), which trigger inflammatory cascades in several cell lineages resulting in altered tissue phenotypes, presenting clinically as psoriatic plaques and, as mentioned, about a quarter develop musculoskeletal problems including synovitis, dactylitis, enthesitis, and axial disease [4-6].

Ustekinumab (UST), a monoclonal antibody that targets the shared p40 subunit of IL12 and IL23, was demonstrated to be highly efficacious and safe in psoriasis. Based on these encouraging results, a phase II study was performed in PsA, followed by two phase III clinical trials, which included both anti-TNF naïve and experienced patients $[7 \bullet, 8 \bullet$. Herein, we will review the pathophysiology of the different manifestations of PsA, present data on the efficacy and safety of UST in PsA, and conclude with a discussion of pipeline agents that target different molecules in the IL23/Th17 pathway.

\section{About IL12 and IL23}

\section{IL23 and psoriatic arthritis}

The central importance of the IL23/Th17 pathway in human disease was initially identified in psoriasis. Injury to the skin by either trauma or infection causes cell 


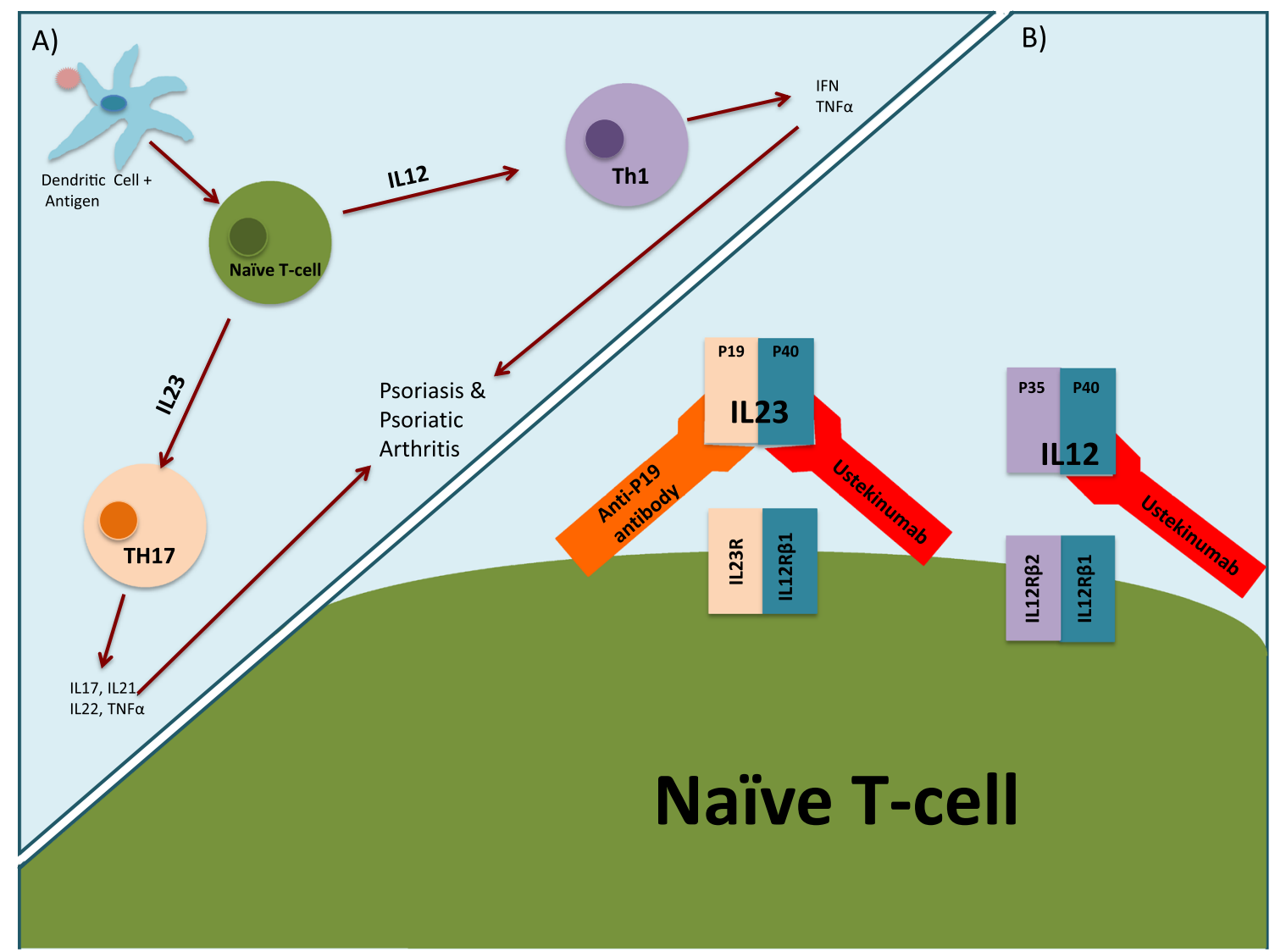

Fig. 1. a Antigen binding to dendritic cells leads to interferon- $\alpha$ mediated activation of naïve T cells. Presence of IL12 leads to the proliferation of Th1 cells, whereas IL23 leads to Th17 development. Th17 cells release IL17, IL21, IL22, and TNF- $\alpha$, which are thought to be the key players in the development of psoriasis and psoriatic arthritis. $\mathbf{b}$ Heterodimeric cytokine IL12 is comprised of p40 and p35 subunits, while IL23 consists of p40 and p19. Ustekinumab is a monoclonal antibody that binds to the p40 subunit common to both IL12 and IL23 and blocks signal transduction through their respective receptors. New anti-p19 antibodies blocks IL23 exclusively. Clinical studies on this new antibody are under way.

death and the release of the antimicrobial cathelicidin LL37 by keratinocytes, which binds intracellular toll-like receptors (TLRs), resulting in the activation of plasmacytoid dendritic cells [3]. These dendritic cells release IFN- $\alpha$, which activates the dermal myeloid dendritic cells and promotes their migration to the lymph nodes [10]. In the lymph node, myeloid dendritic cells secrete IL12 and IL23, which induce differentiation of the naïve T cells into Th1 and Th17 cells, respectively $[2,6,11,12]$. These effector cells then exit the lymph node and re-enter circulation and return to the dermis, a chemotactic process facilitated by expression of surface chemokine receptors CCR4, CCR6, CCR7, and CXCR3 and homing molecules CLA, VLA-4, and LFA-1 [13]. In the skin, Th17 cells secrete cytokines including IL17, IL21, and IL22; the latter found to lead to proliferation of keratinocytes [14]. Likewise, intradermal injection of IL21 has been shown to stimulate human keratinocytes to proliferate and causes epidermal hyperplasia in murine models [15]. This pathologic hyperproliferation of keratinocytes leads to the characteristic thickening of the epidermis observed in psoriasis $[14,16]$. 
Initial clues to the involvement of the IL23/IL17 axis in spondyloarthritis (SpA) arose from the observation that a polymorphism in the receptor for IL23 (IL-23R) was associated with altered susceptibility to ankylosing spondylitis and PsA $[17,18]$. Specifically, R381Q IL23R carriers show decreased IL23dependent IL17 and IL22 production and a lower percentage of circulating Th17 cells [19]. These individuals also showed a decreased IL23-dependent signaling. Interestingly, IL23R R381Q gene carriers are protected against psoriasis, Crohn's disease, and ankylosing spondylitis [20].

Later studies showed that patients with SpA have elevated IL12 and IL23 levels in their synovial fluid and peripheral blood [21]. Elevated IL23 levels have been linked to the presence of human leukocyte antigen B27 (HLA-B27) positivity among these patients [22]. Moreover, in vitro and pre-clinical studies have shown that misfolding in the HLA-B27 protein leads to release of IL23 [22, 23]. IL23, in turn, induces the proliferation of Th17 cells, which then release pro-inflammatory cytokines [24].

The IL23/Th17 axis has been implicated in many of the features that characterize PsA. For example, enthesitis or inflammation at the tendon, ligament, and joint capsule insertion sites is a cardinal feature of PsA. In a recent study, Sherlock et al. [4] showed that administration of IL23 to mice lead to the proliferation of a resident population of innate IL23R+ CD3+ CD4- CD8- T cells at the tendon insertion sites. In turn, enthesitis was associated with increased levels of IL6, IL17, and IL22, which was not observed when mice were pre-treated with anti-IL23 antibody. They concluded that IL23 alone, in the absence of any other inflammatory signal, was sufficient to reproduce the classical enthesitis feature of PsA. The presence of entheseal resident T cells, which expresses the ROR $\gamma t$ transcription factor and secrete an array of TH17associated cytokines, including IL22, may explain how dysregulation of IL23 results in inflammation and new bone formation at the entheses in the absence of synovitis $[4,18]$.

Another key signal transduction molecule in the T cell development is ZAP70. Mice with an activating mutation in the $\mathrm{SH} 2$ domain of this molecule develop axial and peripheral arthritis, which is suppressed in the absence of IL17. Furthermore, studies in SKG mice (ZAP-70 knockout model) have demonstrated that the development of the joint, bone, entheseal, and dactylitic inflammation in these mice is IL23 mediated [25]. Thus, emerging data in animal models implicate an important role for the IL17/IL23 axis in the pathophysiology of psoriasis, enthesitis, synovitis, and abnormal bone turnover in PsA.

\section{Challenges in the treatment of psoriatic arthritis}

The treatment of PsA has been challenging owing to a lack of high-quality trial data supporting the efficacy and safety of traditional disease-modifying antirheumatic drugs (DMARDs). In particular, studies analyzing the efficacy of oral DMARDs have shown no improvement over placebo in the case of methotrexate $[26,27]$ and small effect sizes for sulfasalazine [28]. One phase IIb study did reveal efficacy for leflunomide for the joints only, but a phase III study was never performed [29]. Likewise, a large, retrospective US database looked at patients treated with DMARDs $(n=1698)$ vs. biologics $(n=3263)$ over a 4 -year 
period found that $69 \%$ of patients in the DMARD group required a change in therapy. Of these patients, $64 \%$ were switched to a biologic, and $25 \%$ had a therapy add on. Among the patients who were started on a biologic initially $46 \%$ required a therapy change, and of these, $25 \%$ were switched to a different biologic with $7 \%$ having a therapy add on [30•].

Anti-TNF agents have rapidly moved to the center of the treatment paradigm in PsA due to their efficacy for both skin and musculoskeletal involvement based on high-grade evidence from placebo-controlled trials [31]. Despite the enthusiasm for these agents, the long-term efficacy data are not that encouraging. Up to $40-50 \%$ of patients fail to reach the ACR20 outcome measures in most trials due to primary or secondary non-response or are unable to tolerate these agents [32•]. Moreover, the NOR-DMARD Registry, a multicenter observational study in Norway, looked at 439 patients with PsA who were on their first anti-TNF and reported that if a patient had failed to tolerate or respond to an anti-TNF agent, they had a $60-80 \%$ chance of remaining unresponsive after switching to a second anti-TNF agent [32•]. Therefore, these studies highlight the high rate of change in therapies in PsA patients and underscore the need for additional treatment options.

\section{Ustekinumab}

\section{Ustekinumab in psoriasis}

Ustekinumab is a humanized IgG monoclonal antibody that binds to the P40 subunit common to IL12 and IL23 (Fig. 1b). In the initial in vitro studies, human peripheral blood mononuclear cells (PBMCs) from healthy donors were activated in the presence or absence of recombinant human IL12 or IL23, with or without UST. In the presence of UST, the cell cultures showed reduction in the level of the skin homing molecule CLA as well as reduction in the secretion of IFN- $\alpha$, TNF- $\alpha$, IL17, and IL22 secretion. Therefore, it was concluded that UST would both neutralize the IL12/23 pathway and decrease expression of skin homing and activation markers [33]. In another study, x-ray crystallography demonstrated that UST binds with nearly equal affinity to the p40 component of both IL12 and IL23 [34].

The initial utility of UST was studied in psoriasis, starting with pre-clinical studies and followed by six clinical trials that showed UST to be an effective and relatively safe treatment for psoriasis [35].

PHOENIX 1 and PHOENIX 2 were two pivotal phase III trials that examined the efficacy and safety of UST in psoriasis. These clinical trials were designed to assess long-term efficacy and safety of UST compared with placebo. PHOENIX 1 was a parallel double-blind placebo-control (DBPC) study of 766 patients with moderate-to-severe psoriasis. The subjects were randomly assigned to receive 45 or $90 \mathrm{mg}$ of UST at weeks 0,4 , and then every 12 weeks. The control group received placebo at 0 and 4 weeks and were crossed over to receive UST at 12 weeks. At 12 weeks, $67.1 \%$ of patients who received $45 \mathrm{mg}$, $66.4 \%$ of patients who received $90 \mathrm{mg}$, and $3.1 \%$ of those receiving placebo achieved PASI75. They also found that PASI75 response was better maintained at least 1 year in those who were continued on UST vs. those who were withdrawn. 
Rates of adverse events were generally comparable between subjects in the placebo, UST $45 \mathrm{mg}$, and UST $90 \mathrm{mg}$ groups [36].

PHOENIX 2 was a large multicenter, DBPC study of 1230 patients with moderate-to-severe psoriasis who were again assigned to placebo vs. 45 or $90 \mathrm{mg}$ of UST at weeks 0,4 , and then every 12 weeks. The primary endpoint was measured at 12 weeks; $66.7 \%$ of patients receiving $45 \mathrm{mg}$ of UST and $75.6 \%$ of those receiving $90 \mathrm{mg}$ of UST had achieved PASI75, compared with $3.7 \%$ of patients in the placebo group. Therefore, the PHOENIX 2 trial suggested that dosing intensification would improve clinical response rate in patients with incomplete response to initial treatment with UST [37].

Overall, the PHOENIX trials demonstrated impressive long-term efficacy. The ACCEPT trial was another phase III trial, which was a head-to-head comparison of UST with etanercept (an anti-TNF monoclonal antibody) in 903 patients with moderate-to-severe psoriasis, which showed superiority of UST at 12 weeks [38]. These phase II and III studies involved predominantly Caucasian patients. The PEARL trail was a randomized DBPC trial of 121 Taiwanese and Korean patients with moderate-to-severe psoriasis randomized with a treatment with $45 \mathrm{mg}$ of UST vs. placebo. The results showed that the efficacy (and safety) rates in Asian patients were consistent with previous studies [39]. In 2009, the Food and Drug Administration approved its use for adult patients with moderate-to-severe plaque psoriasis in the US, and approval for this indication in Canada and Europe followed shortly thereafter.

\section{Efficacy of ustekinumab in psoriatic arthritis}

Gottlieb et al. published a phase II randomized DBPC and cross over study of UST in 2009. They studied 146 patients with active PsA, with greater than three tender and swollen joints and either an elevated C-reactive protein (CRP) or significant morning stiffness ( $>45 \mathrm{~min}$ ). Patients were also required to have at least one active psoriatic plaque measuring a minimum of $2 \mathrm{~cm}$ in diameter. About $25 \%$ of the subjects had previously been treated with an anti-TNF agent for PsA. Subjects in the UST arm received $63 \mathrm{mg}$ of the drug at weeks $0,1,2$, and 3 followed by placebo at weeks 12 and 16 . The subjects in the control group received placebo for the first four doses and UST at weeks 12 and 16 . The primary endpoint was the percentage of patients who achieved an American College of Rheumatology (ACR) 20 improvement at 12 weeks. They found that $42 \%$ of the patients in the active group achieved an ACR2 0 at 12 weeks, compared with only $14 \%$ of patients in the control group (Table 1 ). At 36 week follow-up, having received two doses of UST, $45 \%$ of patients in the control group achieved ACR20 [40]. Patients in this study also demonstrated a statistically significant improvement in their quality of life as measured by a $59 \%$ improvement in the measured Dermatology Life Quality Index (DLQI) [41].

Based on the efficacy in the phase IIb trial, two multicenter DBRPC trials of UST in PsA known as PSUMMIT 1 and PSUMMIT 2 were initiated. PSUMMIT 1 recruited 615 patients naïve to anti-TNF therapy. Patients on DMARDs or NSAI $D$ therapy were included in the study. All subjects selected met the Classification Criteria for Psoriatic Arthritis (CASPAR) Criteria for PsA and had active disease based on presence of at least five swollen joints and at least five tender joints. They were randomized 1:1:1 to receive UST of $45 \mathrm{mg}$, $90 \mathrm{mg}$, or placebo at 0,4 , 


\begin{tabular}{|c|c|c|c|c|c|c|}
\hline Trial & $\begin{array}{l}\text { Number of } \\
\text { subjects }\end{array}$ & $\begin{array}{l}\text { TNF } \\
\text { exposure }\end{array}$ & $\begin{array}{l}1^{\circ} \text { Outcome } \\
\text { ACR20/PBO } \% \\
45 / 90 \\
\mathrm{mg} / \mathrm{PBO}^{\mathrm{a}}\end{array}$ & $\begin{array}{l}2^{\circ} \text { Outcome } \\
\text { ACR50 } 170 \% \\
45 / 90 \mathrm{mg}\end{array}$ & $\begin{array}{l}\text { PASI } \\
75 \% \\
45 / 90 \\
\mathrm{mg} / \mathrm{PBO}\end{array}$ & Comments \\
\hline $\begin{array}{l}\text { Gottlieb } \\
\text { et al. [40] }\end{array}$ & 146 & $\begin{array}{l}25 \% \\
\quad \text { experienced }\end{array}$ & 42/14 (PBO) & - & $52 / 5$ & $\begin{array}{l}\text { Altered dosing } \\
\text { regimen }^{\text {a }}\end{array}$ \\
\hline $\begin{array}{l}\text { PSUMMIT } 1 \\
\text { [8] }\end{array}$ & 615 & TNF naïve & $42 / 50 / 23$ & $25 / 12$ & $\begin{array}{c}57 / 62 / \\
11\end{array}$ & $\begin{array}{c}\text { Combined datasets } \\
\text { from PSUMMIT } 1\end{array}$ \\
\hline $\begin{array}{l}\text { PSUMMIT } 2 \\
\text { [7] }\end{array}$ & 312 & $\begin{array}{l}\text { TNF } \\
\text { experienced } \\
\text { TNF naïve }\end{array}$ & $\begin{array}{l}37 / 35 / 15 \\
54 / 55 / 29\end{array}$ & $18 / 6.88$ & $51 / 56 / 5$ & $\begin{array}{l}\text { and } 2 \text { showed } \\
\text { inhibition of } x \text {-ray } \\
\text { progression }\end{array}$ \\
\hline
\end{tabular}

and 12 weeks. In this study, nearly $50 \%$ of patients were on concomitant methotrexate. Patients were eligible for early escape at week 16 if they demonstrated $<5 \%$ improvement in total or swollen joint and the dose of UST was increased (placebo to $45 \mathrm{mg}$ UST and 45 to $90 \mathrm{mg}$ of UST, $90 \mathrm{mg}$ UST continued this dose). Any patients who remained in the placebo group at week 24 crossed over to receive $45 \mathrm{mg}$ of UST, which was continued at week 28 and

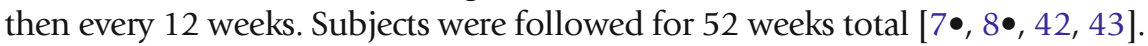

The primary endpoint was ACR20 response at 24 weeks, but the authors also examined ACR 50/70, Disease Activity Score (DAS) 28 using CRP (DAS28-CRP) PASI 75 response Health Assessment Questionnaire-Disability Index (HAQ-DI), and percentage of change in enthesitis and dactylitis scores as secondary measures. Both UST 45 and $90 \mathrm{mg}$ showed a statistically significant improvement in ACR20 response compared to placebo at 24 weeks, and this response difference was maintained at 52 weeks. Likewise, compared with placebo, treatment with either dose of UST resulted in a significantly higher proportion of patients with DAS28-CRP scores less than 2.6 at week 24. Of the patients who had dactylitis at baseline, patients on UST had significantly greater improvement in their dactylitis and enthesitis scores at 24 weeks, compared with placebo. Moreover, the HAQ-DI change from baseline was -0.3 in both treatment groups, compared with 0 in the placebo group. No unexpected adverse reactions were observed in this study. Therefore, PSUMMIT 1 demonstrated that IL12/23 blockade signifi-

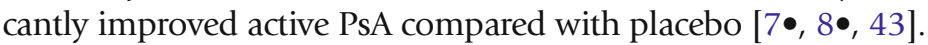

One of the difficulties with the utility of the results from the PSUMMIT 1 trial was that it only included anti-TNF naïve subjects and in clinical practice, most patients have previously been treated with an anti-TNF agent at some point in their disease history. Therefore, there was a need for a viable treatment option for PsA patients who were inadequate responders or did not tolerate anti-TNF agents. PSUMMIT 2 was a second phase III multicenter DBRPC trial, which aimed to address this necessity.

PSUMMIT 2 enrolled 312 adults with PSA who had at least five tender and five swollen joints and a CRP level greater than $0.3 \mathrm{mg} / \mathrm{dL}$. Of these patients, 180 had previous exposure to anti-TNF treatments, and the remaining 132 were anti-TNF naïve. The study design was otherwise identical to PSUMMIT 1, 
including blinded early escape for patients who failed to show improvement at 16 weeks. The primary endpoint was ACR20 response at week 24 . Secondary endpoints included ACR50/70 response, DAS28-CRP response, PASI75, improvements in enthesitis and dactylitis scores, and improvements in HAQ-DI scores. Forty four percent of patients in the UST 45 and $90 \mathrm{mg}$ achieved ACR20, compared with $20 \%$ of patients in the placebo group. Looking at the secondary endpoints, the patients in both active groups had a statistically significant improvement in DAS28-CRP, PASI 75, and ACR50, with the benefits sustaining at 52 weeks. Interestingly, patients who were anti-TNF naïve responded better to UST than patients who had been previously exposed (Table 1). The authors proposed that while the exact cause for this is not clear, it is conceivable that exposure to biologics alter the natural history of the disease. Therefore, PSUMMIT 2 established the efficacy of UST in a more clinically relevant context and showed no significant adverse events [7•].

A pre-planned follow-up study looked at combined radiographic data from PSUMMIT 1 and PSUMMIT 2 trials (total of 927 patients). The primary endpoint was the change in van der Heijde-Sharp (vdH-S) score from baseline to week 24. This analysis showed that both UST 45 and 90 mg lead to inhibition of radiographic progression of joint damage in PsA at 24 weeks. Follow-up to week 52 demonstrated maintained inhibition of radiographic progression for UST-treated patients, and substantial reduction of progression among initial placebo recipients who started UST at week 16 or week 24 [44•]. When looking at each trial alone, PSUMMIT 1 demonstrated significant radiographic progression, but a statistically significant reduction in x-ray progression was not seen in PSUMMIT 2 alone. This was thought to be related to the smaller sample size and numbers of missing radiographic data in a large proportion of the placebo patients [42].

\section{Safety of ustekinumab in treatment of psoriatic arthritis}

A meta-analysis of the initial phase II and phase III trials of the anti-IL12/23 biologic agents (UST and briakinumab) in psoriasis raised concerns for major adverse cardiovascular events (MACEs) [45]. MACE is a composite endpoint of myocardial infarction, cerebrovascular accident, or cardiovascular death during the placebo-controlled phase of treatment in patients receiving at least one dose of study agent or placebo. Among 3179 subjects, ten MACEs were recorded in the placebo-control phase of these studies, compared with zero events in the placebo group of 1474 patients [45], although the difference was not found to be statistically significant when the agents were looked at individually. Nevertheless, these findings lead to briakinumab eventually being pulled from clinical trials.

In spite of these initial concerns, a 5-year follow-up study of the patients in the PHOENIX 1, 2, and ACCEPT trials did not find a significant increase in the number of MACE events with UST vs. placebo. They looked at a total of 3117 patients followed for a period of 4-5 years- 10,000 patient years. They recorded 40 MACE events in 37 patients, all of whom had at least two independent cardiovascular risk factors. Overall, the rates of stroke and cardiovascular death were $0.03 / 100 \mathrm{PY}$ and $0.07 / 100 \mathrm{PY}$, not statistically significant compared with placebo. There was no dose-dependent difference in the rate of overall risk of adverse events in patients who received $45 \mathrm{vs} .90 \mathrm{mg}$ of UST [46]. The rate of other adverse events, including infections and malignancy were not statistically 
significant for either dose. Therefore, the pooled long-term analysis of patients in the three phase III studies of UST in psoriasis did not show evidence of increased risk with dose or duration of therapy. Likewise, the PSUMMIT 1 and 2 trials showed no significant increased risk of infection or malignancy in patients treated with either dose of UST compared with placebo. No MACE events were observed through 16 weeks (the placebo-control phase) [7•, $8 \bullet$. Therefore, the information available to date shows that UST is not only efficacious but also safe in the treatment of PsA.

\section{P19 component: a new target for blockade of IL23}

As mentioned previously, IL12 and IL23 are heterodimeric cytokines with a common subunit (p40), which is targeted by UST. Studies in mice have demonstrated an increased IL23p19 subunit expression in psoriatic lesions, but no increase in the expression of the IL12p35 subunit [47]. These findings kindled interest in the concept that blockade of IL23 may be the more relevant target in psoriasis than IL12. Furthermore, IL23 has been shown to promote greater keratinocyte proliferation than IL12 [48]. In a phase I study, Nakajima et al. showed that treatment of mice with psoriatic plaques with anti-IL23p19 antibodies greatly inhibited epidermal hyperplasia [49]. Based on these results, new humanized IgG monoclonal antibodies (BI655066 [50], guselkumab and tildrakizumab [51] formerly SCH900222 [52]), targeting the p19 subunit of IL23, were developed by several different companies and are currently in phase II clinical studies (Table 2).

The initial trials using these agents in psoriasis have proven promising [51, 53]. It remains to be seen whether these agents will be useful in the treatment of PsA as well as plaque psoriasis. Furthermore, the efficacy of IL23 blockade alone will need to be studied. All the studies with UST have benefited from the blockade of both IL12 and IL23. It is not known to what extent the blockade of IL12 is responsible for the clinical improvement seen in patients treated with UST.

\section{IL17 blockade}

While here, we have focused on IL12 and IL23 inhibition, several noteworthy drugs in the commercial pipelines for PsA focus on IL17 blockade. IL17 inhibitors including secukinumab [54] and ixekizumab [55, 56], and the IL17 receptor

\section{Table 2. Pipeline biologic agents for treatment of psoriatic arthritis}

\begin{tabular}{lll}
\hline Agent & Target & Stage \\
Ustekinumab & P40 subunit of IL12 and IL23 & FDA approved \\
Secukinumab & IL17A & Phase III \\
Ixekizumab & IL17A & Phase III \\
Brodalumab & IL17 receptor & Phase III \\
Guselkumab & P19 subunit of IL23 & Phase II \\
Tildrakizumab (MK-322) & P19 subunit of IL23 & Phase II \\
BI 655066 & P19 subunit of IL23 & Phase II \\
IL interleukin & &
\end{tabular}




\section{IL22 blockade}

inhibitor brodalumab $[57,58 \bullet$ have proven efficacious in the treatment of psoriasis. Results from two phase III studies (FUTURE 1 and FUTURE 2) showed secukinumab to be superior to placebo in the treatment of PsA [59, 60•]. Likewise, a recent phase II trial looking at brodalumab in the treatment of PsA showed efficacy and safety compared with placebo [61]. A multicenter phase III trial of brodalumab in PsA is currently under way [62]. Therefore, drugs that target IL17 and its receptor are an important part of the future armament against PsA.

As described earlier, Th17 cells secrete several pro-inflammatory cytokines that are important in the development of psoriasis. One of these cytokines is IL22, which is thought to cause hyperproliferation of keratinocytes, leading to the characteristic hyperkeratosis seen in PsA [63]. Serum IL22 levels have even been found to correlate with the disease severity of psoriasis patients [64]. Fezakinumab (formerly ILV-094) is a humanized monoclonal IgG antibody against IL-22, which progressed as far as phase II trials, but the data was not released [65].

\section{Compliance with Ethics Guidelines}

\section{Conflict of Interest}

Mahta Mortezavi declares no conflict of interest.

Christopher Ritchlin has consulted for Abbvie, UCB, Regeneron, Sanofi, and Amgen.

\section{Human and Animal Rights and Informed Consent}

This article does not contain any studies with human or animal subjects performed by any of the authors.

\section{References and Recommended Reading}

Papers of particular interest, published recently, have been

highlighted as:

- Of importance

1. Ibrahim G, Waxman R, Helliwell PS. The prevalence of psoriatic arthritis in people with psoriasis. Arthritis Rheum. 2009;61(10):1373-8.

2. Lowes MA, Kikuchi T, Fuentes-Duculan J, et al. Psoriasis vulgaris lesions contain discrete populations of Th1 and Th17 T cells. J Investig Dermatol. 2008;128(5):1207-11.

3. Lowes MA, Suarez-Farinas M, Krueger JG. Immunology of psoriasis. Annu Rev Immunol. 2014;32:227-55.

4. Sherlock JP, Joyce-Shaikh B, Turner SP, et al. IL-23 induces spondyloarthropathy by acting on RORgammat(+) CD3(+)CD4(-)CD8(-) entheseal resident T cells. Nat Med. 2012;18(7):1069-76.

5. Sato K, Suematsu A, Okamoto K, et al. Th17 functions as an osteoclastogenic helper $\mathrm{T}$ cell subset that links $\mathrm{T}$ cell activation and bone destruction. J Exp Med. 2006;203(12):2673-82.
6. Di Meglio P, Nestle FO. The role of IL-23 in the immunopathogenesis of psoriasis. F1000 Biol Rep. 2010;2. doi:10.3410/B2-40.

7. $\quad$ Ritchlin C, Rahman P, Kavanaugh A, et al. Efficacy and safety of the anti-IL-12/23 p40 monoclonal antibody, ustekinumab, in patients with active psoriatic arthritis despite conventional non-biological and biological anti-tumour necrosis factor therapy: 6-month and 1year results of the phase 3, multicentre, double-blind, placebo-controlled, randomised PSUMMIT 2 trial. Ann Rheum Dis. 2014;73(6):990-9.

Shows that PsA patients who are anti-TNF naive had better response to UST than patients who had been previously exposed to biologics.

8. McInnes IB, Kavanaugh A, Gottlieb AB, et al. Efficacy and safety of ustekinumab in patients with active psoriatic arthritis: 1 year results of the phase 3, multicentre, 
double-blind, placebo-controlled PSUMMIT 1 trial. Lancet. 2013;382(9894):780-9.

Ustekinumab significantly improved psoriatic arthritis compared with placebo.

9. Vignali DA, Kuchroo VK. IL-12 family cytokines: immunological playmakers. Nat Immunol.

2012;13(8):722-8.

10. Gunderson AJ, Mohammed J, Horvath FJ, et al. CD8(+) $\mathrm{T}$ cells mediate RAS-induced psoriasis-like skin inflammation through IFN-gamma. J Investig Dermatol. 2013;133(4):955-63.

11. Di Cesare A, Di Meglio P, Nestle FO. The IL-23/Th17 axis in the immunopathogenesis of psoriasis. I Investig Dermatol. 2009;129(6):1339-50.

12. Zaba LC, Fuentes-Duculan J, Eungdamrong NJ, et al. Psoriasis is characterized by accumulation of immunostimulatory and Th1/Th17 cell-polarizing myeloid dendritic cells. J Investig Dermatol. 2009;129(1):79-88.

13. Teraki $Y$, Miyake A, Takebayashi $R$, et al. Homing receptor and chemokine receptor on intraepidermal $\mathrm{T}$ cells in psoriasis vulgaris. Clin Exp Dermatol. 2004;29(6):658-63.

14. Nestle FO, Kaplan DH, Barker J. Psoriasis. N Engl J Med. 2009;361(5):496-509.

15. Caruso R, Botti E, Sarra M, et al. Involvement of interleukin-21 in the epidermal hyperplasia of psoriasis. Nat Med. 2009;15(9):1013-5.

16. Leipe J, Grunke M, Dechant C, et al. Role of Th17 cells in human autoimmune arthritis. Arthritis Rheum. 2010;62(10):2876-85.

17. Rahman P, Inman RD, Maksymowych WP, et al. Association of interleukin 23 receptor variants with psoriatic arthritis. J Rheumatol. 2009;36(1):137-40.

18. Fitzgerald O, Winchester R. Editorial: emerging evidence for critical involvement of the interleukin-17 pathway in both psoriasis and psoriatic arthritis. Arthritis Rheumatol. 2014;66(5):1077-80.

19. Sarin R, Wu X, Abraham C. Inflammatory disease protective R381Q IL23 receptor polymorphism results in decreased primary CD4+ and CD8+ human T-cell functional responses. Proc Natl Acad Sci U S A. 2011;108(23):9560-5.

20. Di Meglio P, Di Cesare A, Laggner U, et al. The IL23R R381Q gene variant protects against immunemediated diseases by impairing IL-23-induced Th17 effector response in humans. PLoS One. 2011;6(2):e17160.

21. Wendling D, Cedoz JP, Racadot E. Serum and synovial fluid levels of p40 IL12/23 in spondyloarthropathy patients. Clin Rheumatol. 2009;28(2):187-90.

22. DeLay ML, Turner MJ, Klenk EI, et al. HLA-B27 misfolding and the unfolded protein response augment interleukin-23 production and are associated with Th17 activation in transgenic rats. Arthritis Rheum. 2009;60(9):2633-43.
23. Colbert RA, DeLay ML, Klenk EI, et al. From HLA-B27 to spondyloarthritis: a journey through the ER. Immunol Rev. 2010;233(1):181-202.

24. Smith JA, Colbert RA. Review: the interleukin-23/interleukin-17 axis in spondyloarthritis pathogenesis: Th17 and beyond. Arthritis Rheumatol. 2014;66(2):231-41.

25. Benham H, Rehaume LM, Hasnain SZ, et al. Interleukin-23 mediates the intestinal response to microbial beta-1,3-glucan and the development of spondyloarthritis pathology in SKG mice. Arthritis Rheumatol. 2014;66(7):1755-67.

26. Kingsley GH, Kowalczyk A, Taylor H, et al. A randomized placebo-controlled trial of methotrexate in psoriatic arthritis. Rheumatology (Oxford).

2012;51(8):1368-77.

27. Baranauskaite A, Raffayová H, Kungurov NV, et al. Infliximab plus methotrexate is superior to methotrexate alone in the treatment of psoriatic arthritis in methotrexate-naive patients: the RESPOND study. Ann Rheum Dis. 2012;71(4):541-8.

28. Gupta AK, Grober JS, Hamilton TA, et al. Sulfasalazine therapy for psoriatic arthritis: a double blind, placebo controlled trial. J Rheumatol. 1995;22(5):894-8.

29. Kaltwasser JP, Nash P, Gladman D, et al. Efficacy and safety of leflunomide in the treatment of psoriatic arthritis and psoriasis: a multinational, double-blind, randomized, placebo-controlled clinical trial. Arthritis Rheum. 2004;50(6):1939-50.

30. Zhang HF, Gauthier G, Hiscock R, et al. Treatment patterns in psoriatic arthritis patients newly initiated on oral nonbiologic or biologic disease-modifying antirheumatic drugs. Arthritis Res Ther. 2014;16(4):420. Study suggests that PsA patients do not remain on the index biologics or DMARDS for a long period of time and most require a trial of several different agents.

31. Gossec L, Smolen JS, Gaujoux-Viala C, et al. European League against rheumatism recommendations for the management of psoriatic arthritis with pharmacological therapies. Ann Rheum Dis. 2012;71(1):4-12.

32.• Fagerli KM, Lie E, van der Heijde D, et al. Switching between TNF inhibitors in psoriatic arthritis: data from the NOR-DMARD study. Ann Rheum Dis. 2013;72(11):1840-4.

A longitudinal observational study of prescriptions of DMAR Ds and biological agents to patients with inflammatory rheumatic diseases. When selecting for PsA patients, those who had been on a TNF inhibitor and were switched to a second TNF inhibitor agent had significantly poorer responses compared with non-switchers.

33. Reddy M, Davis C, Wong J, et al. Modulation of CLA, IL-12R, CD40L, and IL-2Ralpha expression and inhibition of IL-12- and IL-23-induced cytokine secretion by CNTO 1275. Cell Immunol. 2007;247(1):1-11.

34. Luo J, Wu SJ, Lacy ER, et al. Structural basis for the dual recognition of IL-12 and IL-23 by ustekinumab. J Mol Biol. 2010;402(5):797-812.

35. Hong K, Chu A, Lúdvíksson BR, et al. IL-12, independently of IFN-gamma, plays a crucial role in the 
pathogenesis of a murine psoriasis-like skin disorder. J Immunol. 1999;162(12):7480-91.

36. Leonardi CL, Kimball AB, Papp KA, et al. Efficacy and safety of ustekinumab, a human interleukin-12/23 monoclonal antibody, in patients with psoriasis: 76week results from a randomised, double-blind, placebo-controlled trial (PHOENIX 1). Lancet. 2008;371(9625):1665-74.

37. Papp KA, Langley RG, Lebwohl M, et al. Efficacy and safety of ustekinumab, a human interleukin-12/23 monoclonal antibody, in patients with psoriasis: $52-$ week results from a randomised, double-blind, placebo-controlled trial (PHOENIX 2). Lancet. 2008;371(9625):1675-84.

38. Griffiths CE, Strober BE, van de Kerkhof P, et al. Comparison of ustekinumab and etanercept for moderate-to-severe psoriasis. N Engl J Med. 2010;362(2):118-28.

39. Tsai TF, Ho JC, Song M, et al. Efficacy and safety of ustekinumab for the treatment of moderate-to-severe psoriasis: a phase III, randomized, placebo-controlled trial in Taiwanese and Korean patients (PEARL). J Dermatol Sci. 2011;63(3):154-63.

40. Gottlieb A, Menter A, Mendelsohn A, et al. Ustekinumab, a human interleukin 12/23 monoclonal antibody, for psoriatic arthritis: randomised, doubleblind, placebo-controlled, crossover trial. Lancet. 2009;373(9664):633-40.

41. Kavanaugh A, Menter A, Mendelsohn A, et al. Effect of ustekinumab on physical function and health-related quality of life in patients with psoriatic arthritis: a randomized, placebo-controlled, phase II trial. Curr Med Res Opin. 2010;26(10):2385-92.

42. Weitz JE, Ritchlin CT. Ustekinumab: targeting the IL-17 pathway to improve outcomes in psoriatic arthritis. Expert Opin Biol Ther. 2014;14(4):515-26.

43. Gottlieb A, Narang K. Ustekinumab in the treatment of psoriatic arthritis: latest findings and clinical potential. Ther Adv Musculoskelet Dis. 2013;5(5):277-85.

44. Kavanaugh A, Ritchlin C, Rahman P, et al. Ustekinumab, an anti-IL-12/23 p40 monoclonal antibody, inhibits radiographic progression in patients with active psoriatic arthritis: results of an integrated analysis of radiographic data from the phase 3, multicentre, randomised, double-blind, placebocontrolled PSUMMIT-1 and PSUMMIT-2 trials. Ann Rheum Dis. 2014;73(6):1000-6.

Treatment with Ustekinumab at both 45 and $90 \mathrm{mg}$ doses significantly inhibited radiographic progression of joint damage in patients with active PsA.

45. Ryan C, Leonardi CL, Krueger JG, et al. Association between biologic therapies for chronic plaque psoriasis and cardiovascular events: a metaanalysis of randomized controlled trials. JAMA. 2011;306(8):864-71.

46. Papp KA, Griffiths CE, Gordon K, et al. Long-term safety of ustekinumab in patients with moderate-tosevere psoriasis: final results from 5 years of follow-up. Br J Dermatol. 2013;168(4):844-54.
47. Lee E, Trepicchio WL, Oestreicher JL, et al. Increased expression of interleukin 23 p19 and p40 in lesional skin of patients with psoriasis vulgaris. J Exp Med. 2004;199(1):125-30.

48. Aggarwal S, Ghilardi N, Xie $\mathrm{MH}$, et al. Interleukin-23 promotes a distinct CD4 T cell activation state characterized by the production of interleukin-17. J Biol Chem. 2003;278(3):1910-4.

49. Nakajima K, Kanda T, Takaishi M, et al. Distinct roles of IL-23 and IL-17 in the development of psoriasis-like lesions in a mouse model. J Immunol. 2011;186(7):4481-9.

50. Boehringer-Ingelheim. BI 655066 dose ranging in psoriasis, active comparator ustekinumab. 2015 1/22/ 2015; Available from: https://clinicaltrials.gov/ct2/ show/NCT02054481.

51. Sofen H, Smith S, Matheson RT, et al. Guselkumab (an IL-23-specific mAb) demonstrates clinical and molecular response in patients with moderate-to-severe psoriasis. J Allergy Clin Immunol. 2014;133(4):1032-40.

52. Zandvliet A, Glasgow S, Horowitz A, et al. Tildrakizumab, a novel anti-IL-23 monoclonal antibody, is unaffected by ethnic variability in Caucasian, Chinese, and Japanese subjects. Int J Clin Pharmacol Ther. 2015;53(2):139-46.

53. Jancin B. Novel psoriasis biologic wows with jawdropping results. 2014. Available from: http://www. rheumatologynews.com/index.php?id=8929\&type= 98\&tx_ttnews\%5Btt_news\%5D=308521\&cHash= da03e20e36.

54. Ohtsuki M, Morita A, Abe M, et al. Secukinumab efficacy and safety in Japanese patients with moderate-tosevere plaque psoriasis: subanalysis from ERASURE, a randomized, placebo-controlled, phase 3 study. J Dermatol. 2014;41(12):1039-46.

55. Saeki H, Nakagawa H, Ishii T, et al. Efficacy and safety of open-label ixekizumab treatment in Japanese patients with moderate-to-severe plaque psoriasis, erythrodermic psoriasis and generalized pustular psoriasis. J Eur Acad Dermatol Venereol. 2014. doi:10. $1111 /$ jdv. 12773.

56. Company E.L.A. A study of ixekizumab in participants with active psoriatic arthritis (SPIRIT-P1). 2015 1/13/ 2015; Available from: https://clinicaltrials.gov/ct2/ show/NCT01695239.

57. Papp K, Menter A, Strober B, et al. Efficacy and safety of brodalumab in subpopulations of patients with difficult-to-treat moderate-to-severe plaque psoriasis. J Am Acad Dermatol. 2014;72(3):436-439.e1.

58. Papp K, Leonardi C, Menter A, et al. Safety and efficacy of brodalumab for psoriasis after 120 weeks of treatment. J Am Acad Dermatol. 2014;71(6):1183-1190 e3.

Brodalumab demonstrated safety and efficacy through 120 weeks in patients with moderate to severe plaque psoriasis. The agent is now in Phase III trial for treatment of PsA.

59. Communications N.G. Novartis AIN457 (secukinumab) is the first ever IL-17A inhibitor to meet primary endpoint in two phase III studies in psoriatic 
arthritis. 09/25/2014, Novartis Global

Communications: http://hugin.info/134323/R/

1858095/650696.pdf.

60. McInnes IB, Mease PJ, Kirkham B, et al. Secukinumab, a human anti-interleukin-17A monoclonal antibody, improves active psoriatic arthritis: 24-week efficacy and safety data from a phase 3 randomized, multicenter, double-blind, placebo-controlled study using subcutaneous dosing. 11/18/2014, ACR late-breaking abstract oral session.

Secukinumab, Ixekizumab, and Brodalumab are amongst the emerging anti-IL 17 agents with promising efficacy and safety in the treatment of psoriasis and now psoriatic arthritis.

61. Mease PJ, Genovese MC, Greenwald MW, et al.

Brodalumab, an anti-IL17RA monoclonal antibody, in psoriatic arthritis. N Engl J Med. 2014;370(24):2295306.

62. Amgen. Study of efficacy and safety of brodalumab in subjects with psoriatic arthritis (AMVISION-2). 2015 1/26/2015]; Available from: https://clinicaltrials.gov/ ct2/show/NCT02024646?term=brodalumab\&rank=4.

63. Krueger JG. Hiding under the skin: a welcome surprise in psoriasis. Nat Med. 2012;18(12):1750-1.

64. Hao JQ. Targeting interleukin-22 in psoriasis. Inflammation. 2014;37(1):94-9.

65. Pfizer. Study to evaluate the safety and efficacy of ILV094 in subjects with rheumatoid arthritis. Available from: https://clinicaltrials.gov/show/NCT00883896. 